\title{
Transfer from Spatial Education to Verbal Reasoning and Prediction of Transfer from Classroom-Based Neural Change
}

Robert A. Cortes ${ }^{1}$, Emily G. Peterson², David J. M. Kraemer ${ }^{3}$, Robert A. Kolvoord ${ }^{4}$, David H. Uttal $^{5}$, Nhi Dinh ${ }^{1,6}$, Adam B. Weinberger ${ }^{1,7}$, Richard J. Daker ${ }^{1}$, Ian M. Lyons ${ }^{1}$, Daniel Goldman ${ }^{1}$, \& Adam E. Green ${ }^{1,8^{*}}$

1Department of Psychology, Georgetown University

${ }^{2}$ School of Education, American University

${ }^{3}$ Department of Education, Dartmouth College

${ }^{4}$ College of Integrated Science and Engineering, James Madison University

${ }^{5}$ Department of Psychology, Northwestern University

${ }^{6}$ Bloomberg School of Public Health, Johns Hopkins University

${ }^{7}$ Center for Neuroaesthetics, University of Pennsylvania

8Interdisciplinary Program in Neuroscience, Georgetown University

*Address correspondence to AEG

Email: aeg58@georgetown.edu

Author Contributions: Conceptualization: A.E.G., R.A.K, and D.H.U.; Methodology: D.H.U, A.E.G, E.G.P., D.J.M.K., R.A.C., and I.M.L.; Investigation: E.G.P, N.D., D.G., R.A.C., R.J.D., and A.B.W.; Resources: A.E.G., R.A.K; Writing original draft: A.E.G. and R.A.C.; Writing - review \& editing: D.J.M.K, E.G.P, R.A.K., N.D., D.G., R.A.C., R.J.D., A.B.W., and D.H.U.; Visualizations: R.A.C. and A.B.W.; Supervision: A.E.G., R.A.K., and D.H.U.

Competing Interest Statement: The authors declare no competing interests.

Keywords: Spatial, Education, Reasoning, Transfer, Neuroimaging 


\title{
PREPRINT OF UNPUBLISHED MANUSCRIPT
}

\begin{abstract}
Assessing whether learning in one domain is transferable to abilities in other domains often eludes traditional testing. Thus, a question with bearing on the promise of neuroscience for education is whether neural changes that accompany in-school curriculum learning can improve prediction of learning transfer. Separately, debate in philosophy and psychology has long concerned whether spatial processes underlie seemingly nonspatial/verbal human reasoning (e.g., mental model theory; MMT). If so, education that fosters spatial cognition might yield transfer to improved verbal reasoning. Here, in real-world classrooms studied in a quasiexperimental design, a STEM curriculum devised to foster spatial cognition yielded improved spatial abilities and-consistent with MMT-transferred beyond the spatial domain to improved verbal reasoning. Further supporting MMT, the more students' spatial ability improved, the more their verbal reasoning improved, and spatial ability improvement mediated curriculum transfer. At the neural level, longitudinal fMRI detected curriculum-driven changes in activity, connectivity, and representational similarity of brain regions implicated in spatial cognition. Critically, changes in spatial cognition-linked neural activity robustly predicted curriculum transfer-more accurately than testing and grades-and mediated this transfer. Reports by the National Research Council and others note that spatial abilities reliably predict STEM achievement, but that broad adoption of spatial cognition-focused curricula depends on classroom-based evidence of efficacy and mechanisms-of-change. The present findings support the real-world application of MMT to classrooms via "spatial education." Further, demonstrating that in-school neural change can predict transfer over-and-above performance-based assessment suggests the long-term achievability of neurally-informed curriculum development that leverages neural change to identify and design transferable curricula.
\end{abstract}




\section{PREPRINT OF UNPUBLISHED MANUSCRIPT}

\section{Significance Statement}

Ongoing debate surrounds the promise of neuroscience for education. The present research demonstrates proof-of-principle and real-world classroom achievability for using longitudinal neural change to predict a valuable educational outcome-learning transfer-more accurately than performance-based learning assessment. In parallel, this work tests the educational prediction of a prominent theory of human reasoning-mental model theory (MMT)-in which spatial processes covertly support verbal reasoning. A spatially-enriched STEM curriculum in high schools improved MMT-related spatial ability and yielded verbal-domain transfer to improved reasoning. Longitudinal neuroimaging detected curriculum-related changes in activity, connectivity, and representational similarity in spatial cognition-implicated brain regions, including neural changes that predicted transfer over-and-above performance-based measures. Evidence of real-world efficacy and neurocognitive mechanisms-of-change supports the application of MMT to classrooms via "spatial education." 


\section{PREPRINT OF UNPUBLISHED MANUSCRIPT}

\section{Main Text \\ Introduction}

The promise of neuroscience to support education remains theoretically intriguing but empirically underdeveloped(1-3). A central question is whether brain-imaging can improve traditional performance-based means of assessing teaching and learning. More accurate prediction of learning transfer-predicting when learning a curriculum will generalize to untrained skill domainswould be especially valuable because transferability is often difficult to capture with traditional assessments(4). Other questions concern neuroscience's potential to achieve broad impacts for education given the impracticality of large-scale neuroimaging (we can't scan every student's brain). This may constrain the impacts of approaches intended to assess individual students' learning or individual abilities and needs. However, research that can also support the design and improvement of curricula-assessing the curriculum rather than the student-might achieve broad impacts from relatively small-scale implementations because curricula that are developed at small/local scales can be applied broadly(5-7). Thus, determining whether neural changes that accompany curriculum learning can be leveraged to evaluate the efficacy of curricula (e.g., using neural changes to help identify curricula that impart transferable learning), has the potential to support broader impacts. Detecting neural changes associated with real-world curriculum learning requires pairing longitudinal neural measurement with a specific in-school curriculum of interest. To date, longitudinal neuroimaging is usually paired with laboratory-based interventions and/or intensive training, rather than ecologically valid K-12 curricula in real-world classrooms. However, insightful work on $1^{\text {st }}$ grade attendance/exposure and an intensive LSAT preparation course in college classrooms $(8)$, and neuroimaging during lectures viewed remotely by college students outside the classroom(9) suggest the potential efficacy of longitudinal fMRI to detect curriculumdriven neural change in real-world K-12 classroom environments.

A longer-standing question in philosophy and psychology concerns the extent to which spatial cognitive processes might covertly underlie seemingly verbal forms of human cognition $(10,11)$. Mental model theory (MMT) posits that ostensibly verbal information is processed by co-opted neural resources that evolved to support visuo-spatial operations in primates $(10,12)$. If this is correct, fostering spatial processing could yield transfer to improved performance beyond the spatial domain. MMT specifically posits that the spatial process of "scanning"-inspecting spatial representations of information for key features-supports mental model-based reasoning(9; e.g., scanning spatial representations of verbal reasoning premises to determine whether they align to validate a logical conclusion). This leads to the educational hypothesis that curricula designed to foster spatial cognition-especially curricula that develop spatial scanning ability-might yield transfer to cognitive abilities supported by mental modeling beyond the spatial domain, especially verbal reasoning. Behavioral and neuroimaging evidence indicates that verbal deductive reasoning often elicits spatial representations and engages spatially-implicated brain regions (especially posterior parietal cortex; PPC;12-14). However, beyond the binary question of whether spatial resources are engaged, MMT suggests that educational improvement is likely to depend on developing the effectiveness of these resources (i.e., behavioral and neural indicators of improved spatial processing), and their integration with "executive" resources that guide reasoning (e.g., functional connectivity of PPC to dorsolateral prefrontal cortex [DLPFC] during reasoning; 12, 15-18).

These broader questions inform a timely decision for educators and policy makers concerning whether schools should adopt "spatial education" (i.e., classroom curricula devised to integrate visualization tools and spatial problem-solving strategies in order to develop spatial thinking;19, 20). Individual differences in spatial ability robustly predict STEM achievement(19-23), however implementations of curricula designed to bolster spatial ability remain rare in real-world classrooms $(19,20,23)$. This scarcity of school-based spatial education has made it difficult to study outcomes. Reports from the National Research Council (NRC;19), the Organisation for Economic 


\section{PREPRINT OF UNPUBLISHED MANUSCRIPT}

Cooperation and Development (OECD;20), and American Enterprise Institute(AEI;23) indicate that, despite great theoretical promise to support STEM achievement, wider in-school adoption of spatial education is likely to depend on empirically demonstrating efficacy and mechanisms-of-change for spatial curricula implemented in real-world classroom settings.

Here, in real-world high school STEM classrooms, we tested the prediction that a spatial curriculum would yield improved spatial abilities, and whether the curriculum would transfer beyond the spatial domain to verbal reasoning. Students who received the spatial curriculum were compared to propensity scoring-matched control students at the same schools. To further test the educational hypothesis of MMT, we investigated whether improvement in spatial abilities predicted improvement in verbal reasoning. Employing $\mathrm{fMRI}$ to test for longitudinal neural changes in verbal reasoning, we predicted that the spatial curriculum would be associated with changes in activity, connectivity, and representational similarity in brain regions implicated in spatial cognition. We further investigated whether neural changes associated with the spatial curriculum were related to improvement in verbal reasoning. For example, based on the MMT claim that reasoning is supported by the integration of spatial neural resources with executive function resources $(12,15-18)$, we predicted that improvement on verbal reasoning in the spatial curriculum would be associated with increased functional connectivity of spatial cognitionimplicated brain regions (including PPC) to a DLPFC region that is meta-analytically implicated in verbal reasoning. Next, we tested whether the spatial curriculum was associated with longitudinal neural changes during a measure of spatial scanning ability. We then tested the predictions that curriculum-related neural changes on this spatial scanning task were associated with improvement in spatial scanning performance, and (mirroring the behavioral analysis of spatial scanning) that these changes also predicted improvement in verbal reasoning performance. Finally, in order to compare the predictive value of neural change vs. performance assessment for predicting learning transfer, we tested whether neural changes observed during the spatial scanning task predicted transfer to verbal reasoning over-and-above performance on the same spatial scanning task and over-and-above performance on a broader set of academic and cognitive assessments.

Spatial education was studied in five public high schools in northern Virginia that adopted a spatially-enriched Geoscience curriculum(24) in which students construct and evaluate spatial representations (maps) of real-world geographical datasets using Geographic Information Systems technology (SI Materials and Methods; Fig. S1). The course, first adopted by a group of Virginia high schools in 2005, is named, "The Geospatial Semester," though it is now implemented over two semesters. A central component of the curriculum-designed by R.A.K. and classroom educators-is to develop students' ability to scan maps to identify key spatial features, and determine how these features are related across maps (e.g., across different times or places; $S I$ Materials and Methods; Fig. S1;24). The curriculum is also intended to foster more frequent use of spatial thinking strategies.

In 346 students (206 female, 140 male; mean age = 16.61 years), propensity scoring methods were employed using linear combinations of background variables including experience, interests, demographics, and academic performance ( $S I$ Materials and Methods) to match Geospatial students with Controls on their overall 'propensity' to enroll in the Geospatial course $(25,26)$. Propensity scoring methods reduce selection bias in real-world experimental paradigms for which randomization-to-groups is impossible(25). Participants in the main study $(N=182)$, comprising Geospatial ( $n=77,32$ female, 16.66 years) and Control ( $n=105,56$ female, 16.63 years) groups, were tested in the summers before (T1) and after (T2) the school-year on measures of verbal reasoning(12), spatial scanning(27), and mental rotation(28; Fig. 1). Sixty-three participants performed these tasks during fMRI (Geospatial $n=32$, 13 female, 16.36 years; Control $n=31 ; 18$ female; 16.60 years). The primary performance outcome was correct responses per second(29; $S I$ Materials and Methods). Participants also completed a survey measure of spatial "habits-ofmind"(30) before and after the school-year. Geospatial and Control groups (and fMRI sub-groups) 


\section{PREPRINT OF UNPUBLISHED MANUSCRIPT}

did not differ significantly on any task at T1. Likewise, the final Geospatial and Control groups (and fMRI sub-groups) were equated on all background variables that predicted Geospatial enrollment and other demographic, academic, and spatial ability variables (Table S1), indicating that propensity score matching was successful(31).

\section{Results}

\section{Assessing spatial learning and verbal-domain transfer}

We tested whether participation in the Geospatial curriculum yielded improvement on behavioral measures via Group(Geospatial, Control)-by-Time(T1, T2) ANOVAs covarying academic ability (PSAT), scholastic performance (GPA), and Gender(32) in the full main study sample $(N=182)$.

To test the MMT-based hypothesis of transfer from spatial education to verbal reasoning, participants completed a syllogistic deductive verbal reasoning task (Reasoning) modified from Ruff et al.(33). MMT research has strongly indicated that syllogistic deduction is supported by mental modeling(34). On each trial, participants read two premises and determined whether a subsequent conclusion was valid based on the premises (Fig. 1b). Half of the trials included words that referred to overtly spatial relations (e.g., "The cow is above the pig") and half included words that referred to overtly nonspatial relation trials (e.g., "The cow is better than the pig"). Consistent with the MMTbased prediction of verbal-domain transfer, Geospatial students showed greater Reasoning improvement than Controls from T1 to T2 (Group-by-Time Interaction: $F[4,15]$ ) $=5.60, p=.019$; Fig. 1b; Table S3). Geospatial students also showed greater improvement when nonspatial relation trials (e.g., "better than") were analyzed separately $(F 4,158]=6.65, p=.011$; Table S4), providing additional evidence that transfer extended beyond the spatial domain.

Two measures tested spatial learning outcomes directly related to the Geospatial curriculum (Fig. 1b). The Embedded Figures Task (EFT;23), taken from Walter \& Dassonville(35), was selected to assess spatial scanning because of the theorized role of spatial scanning in supporting mental modeling (12). The Geospatial curriculum aims to develop spatial scanning of maps (e.g., scanning the spatial features represented on a map to determine whether they align with spatial features on another map). In EFT, participants spatially scanned features of complex figures to determine whether simpler target figures aligned with figures embedded in the complex figures. Consistent with the prediction of improved spatial scanning ability in the spatial curriculum, Geospatial students improved more than Controls on EFT $(F 4,158]=5.38, P=.022$; Fig. 1b; Table S2). Although EFT was overtly spatial and measured a skill emphasized by the Geospatial curriculum (2-dimensional spatial scanning), students were not exposed to EFT during the Geospatial course. The curriculumdriven improvement in EFT can thus be considered near transfer. Geospatial students also increased more than Controls in self-reported use of spatial thinking strategies on the Spatial Habits-of-Mind Inventory(27; $F[4,15])=10.27, P=.002$; Fig. 1b; Table S6). The Geospatial curriculum thus achieved two primary learning goals within the spatial domain (improved spatial scanning, and increased spatial habits-of-mind). Additionally, Geospatial course grades were generally high (78.4\% A or A+), indicating strong curriculum learning (SI Materials and Methods).

A third spatial measure, mental rotation (MRT), taken from Shepard \& Metzler(28, 36), was used to assess 3-dimensional spatial rotation of objects. Participants determined whether two images of 3-dimensional objects depicted rotations of the same object. Although 3-dimensional rotation is not strongly related to the content of the Geospatial curriculum, which focuses on 2-dimensional spatial representations (as in EFT), we hypothesized that the Geospatial Course might improve spatial cognition broadly, and MRT was of interest because it is implicated in mental modeling $(10,12)$. However, MRT performance did not show significant curriculum-specific improvement (Group-byTime Interaction: $F[4,158]=1.40, p=.239$; Fig. 1b; Table S5), although Geospatial students improved nominally more than Controls. 


\section{PREPRINT OF UNPUBLISHED MANUSCRIPT}

\section{Testing the educational prediction of mental model theory}

We next investigated the MMT-based hypothesis of spatial curriculum transfer in greater depth. If development of the spatial processes theorized to support mental modeling actually accounted for the transfer to verbal reasoning that we observed, then improved verbal reasoning should be related to improvement in these spatial abilities. We tested this prediction, again controlling for PSAT, GPA, and Gender in all models. Consistent with MMT, longitudinal change in spatial scanning performance on EFT from T1 to T2 $(\triangle \mathrm{EFT})$ predicted change in Reasoning performance from T1 to T2 ( $\Delta$ Reasoning) for all Reasoning trials $(\beta=.288, p<.001$; Table S11), and for nonspatial relation trials (e.g., "better") when analyzed separately $(\beta=.291, p<.001$; Table S12). That is, the more students' spatial scanning improved, the more their verbal reasoning improved. Further, $\triangle E F T$ mediated the transfer association of the spatial curriculum to improved reasoning for all Reasoning trials (Indirect Effect: $p=.028$; Fig. 3 ) and nonspatial trials separately $(p=.036$; Fig. S6), further suggesting that improvement in spatial scanning may have been a cognitive mechanism by which spatial education improved verbal reasoning. Additionally supporting MMT, longitudinal change in mental rotation $(\triangle \mathrm{MRT})$ predicted $\Delta$ Reasoning for all trials $(\beta=.453$, $p<.001$; Table S13) and nonspatial trials separately $(\beta=.522, p<.001$; Table S14). That is, the more students' 3-dimensional rotation improved, the more their verbal reasoning improved. $\triangle \mathrm{MRT}$ was not considered as a mediator because, as noted above, it was not significantly associated with the independent Group variable (Geospatial vs. Control). Simultaneous regression showed that $\triangle \mathrm{EFT}$ and $\triangle \mathrm{MRT}$ each independently predicted $\Delta$ Reasoning (for all Reasoning trials and for nonspatial trials only; all $\beta>.149$, all $p<.034$; Tables S15-16).

\section{Spatial curriculum-related neural changes during verbal reasoning}

Having observed transfer from the spatial curriculum to verbal reasoning performance, we investigated whether this transfer was accompanied by concurrent longitudinal changes at the neural level during the Reasoning task, employing whole-brain Group(Geospatial, Control)-byTime(T1, T2) ANOVA in the $63 \mathrm{fMRI}$ participants. This analysis revealed a Group-by-Time interaction, indicating longitudinal changes in activity during Reasoning that were greater for students who received the Geospatial curriculum than for Control students in two regions of PPC: left anterior intraparietal sulcus (alPS; Fig. 2a), and a cluster extending from left alPS into left superior parietal lobule (alPS-SPL; Table S19). Because we hypothesized that the spatial curriculum would yield neural change in spatial cognition-implicated regions, we masked the wholebrain results with the meta-analytic Neurosynth(37) association test map for the term "spatial" (henceforth, "SpatialMap"). The more anterior IPS cluster overlapped with SpatialMap and was thus included in subsequent analyses. The alPS-SPL cluster did not. alPS activity is strongly implicated in spatial cognition, and especially spatial attention(35, 38-40). No changes were greater for Control than Geospatial within SpatialMap (SI Materials and Methods). We additionally explored whether the effects of the spatial curriculum differed by Gender, but Group-by-Time-byGender ANOVA indicated no 3-way interaction.

MMT posits that spatial cognition resources operate in conjunction with executive function resources to support reasoning(12, 15-18). Prior neuroimaging evidence indicates that spatial cognition-implicated brain regions interact with domain-general reasoning resources centered in DLPFC to support mental model-based reasoning $(12,16)$. Thus, concurrent with our prediction that the spatial curriculum would improve reasoning (as confirmed above), we predicted that the spatial curriculum would be associated with longitudinally increased functional connectivity of DLPFC to SpatialMap during Reasoning. To represent this prediction, we generated a seed region for connectivity analysis using a DLPFC region identified via meta-analysis of the form of verbal reasoning studied here (syllogistic deduction;14). Whole-brain Group-by-Time ANOVA for 


\section{PREPRINT OF UNPUBLISHED MANUSCRIPT}

psychophysiological interaction-based functional connectivity to the DLPFC seed region was then masked with SpatialMap. Consistent with the MMT-based prediction, this analysis revealed that Geospatial students increased more than Controls in connectivity of SpatialMap regions to DLPFC; Fig. 2c; Fig. S10; Table S23). No connectivity changes were greater for Control students than Geospatial students. A Group-by-Time-by-Gender interaction emerged whereby female Geospatial students showed greater curriculum-related increase in connectivity to parietal and premotor regions than males (Fig. S11; Table S24), suggesting that this connectivity increase was especially strongly associated with spatial curriculum-based reasoning improvement in female students.

A pedagogical goal of spatial education is to encourage students to "spatialize" the information they encounter (i.e., developing spatial representations of information even when the information is presented in nonspatial modalities;22, 24). As noted above, the Reasoning task was devised such that half the trials included overtly spatial relations (e.g., "above") and the other half included overtly nonspatial relations (e.g., "better"). A primary reason for this was to enable us to directly test the prediction that the Geospatial curriculum was associated with "spatialized" representation of overtly nonspatial relations. We operationalized this prediction using representational similarity analysis (RSA; SI Materials and Methods). Specifically, we tested whether the neural patterns associated with overtly nonspatial relation trials became more similar to the patterns associated with overtly spatial relation trials in the SpatialMap cluster that showed spatial curriculum-related change in activity during Reasoning (alPS; Fig. 2a), and whether such spatialization was greater for Geospatial relative to Control students. Consistent with these predictions, Geospatial students showed increased similarity between nonspatial and spatial trials in alPS (paired $t$-test for T1 vs. T2 similarity in Geospatial students: $t=3.43, p=.001$; Fig. 2b; SI Materials and Methods). Further analysis of alPS patterns indicated that the change in nonspatial-to-spatial similarity was primarily driven by changes in the nonspatial trials (i.e., nonspatial trials becoming more similar to spatial trials). That is, nonspatial relation trials at $\mathrm{T} 1$ were significantly less similar to nonspatial relation trials at T2 than spatial relation trials at T1 were to spatial relation trials at T2 $(t=2.44, p=.018)$. Group-by-Time ANOVA showed that this increase in nonspatial-to-spatial similarity in alPS was greater in Geospatial students than in Controls $(F 2,61]=4.71, p=.034$; Table S25).

To further investigate potential neural mechanisms-of-change associated with the effect of the spatial curriculum on verbal reasoning, we tested whether each curriculum-related longitudinal neural change we observed for Reasoning was associated with change in Reasoning performance ( $\triangle$ Reasoning). All models again controlled for PSAT, GPA, and Gender. Local change in Reasoning activity in alPS was not significantly associated with change in Reasoning performance $(\beta=.152$, $p=.238$; Table S31). Likewise, RSA-based nonspatial-to-spatial similarity increase within the alPS cluster was not significantly associated with $\Delta$ Reasoning $(\beta=.242, p=.062$; Table S32). However, increased connectivity of SpatialMap to DLPFC was significantly associated with $\triangle$ Reasoning $(\beta=.309, p=.016$; Table S30). That is, the more SpatialMap-to-DLPFC connectivity during Reasoning increased longitudinally, the more students' reasoning improved. Subsequent mediation analyses did not reveal significant mediation of the association between the Geospatial curriculum and improved reasoning (Indirect Effect: $p=.088$ ).

\section{Spatial curriculum-related neural changes during spatial scanning (EFT)}

As described above, the spatial curriculum was associated with improvement in spatial scanning performance on EFT. We thus investigated whether this improvement was accompanied by concurrent curriculum-driven longitudinal changes in neural activity for EFT. Whole-brain Group(Geospatial, Control)-by-Time(T1, T2) ANOVA controlling for PSAT, GPA, and Gender in the $63 \mathrm{fMRI}$ participants identified longitudinal changes that were greater in Geospatial students than Controls in two PPC regions (Fig. 3): left alPS (proximate to, but not overlapping, the change in aIPS during Reasoning) and right inferior parietal lobule (IPL), as well as regions in middle frontal gyrus, frontal pole, and premotor cortex (Fig. S7; Table S18). Because EFT is a spatial cognition 


\section{PREPRINT OF UNPUBLISHED MANUSCRIPT}

task, we did not mask the results with SpatialMap to identify regions implicated in spatial cognition. While all of the regions where longitudinal changes were observed have been associated with spatial function, this evidence is strongest for the PPC regions $(38,41-43)$. No changes in activity were greater for Control students than Geospatial students, and Group-by-Time-by-Gender ANOVA indicated no 3-way interaction. No connectivity or RSA analyses were conducted for EFT because there were no relevant hypotheses. Neuroimaging data for the other spatial ability measure we administered (MRT) were not included for the main analyses that sought to identify neural correlates of spatial curriculum-related change in performance because, as described above, MRT performance was not significantly related to the Geospatial curriculum. fMRI analyses for MRT are reported in SI Materials and Methods (Fig. S9; Tables S21-22).

Paralleling the Reasoning task analyses (above), we next tested whether each of these curriculumrelated changes for EFT neural activity was associated with change in spatial scanning performance $(\triangle \mathrm{EFT})$, again controlling for PSAT, GPA, and Gender. Longitudinal change in each of the observed PPC regions, i.e., change in alPS activity ( $\triangle$ alPS_EFT) and change in IPL activity ( $\Delta \mathrm{IPL}$ EFT), was strongly associated with $\Delta \mathrm{EFT}$ (All $\beta>.424$, all $p<.001$; Tables S26-S27). That is, the more that activity in each of these PPC regions increased longitudinally, the more students improved in spatial scanning performance. The activity changes observed in frontal and premotor regions were not significantly associated with $\Delta \mathrm{EFT}$ (All $\beta<.201$, all $p>.091$ ). IPL and alPS (as noted above), have been consistently implicated in spatial attention(38, 41-43), including the attentional demands of spatial scanning in EFT(35). Notably, these regions also appear to support attentional demands of ostensibly nonspatial tasks (e.g., mental modeling-associated verbal reasoning, numerical cognition, and nonspatial working memory;35, 39, 40, 44).

\section{Using neural change to predict transfer, and comparison of neural change vs. performance- based assessment}

A primary objective of this research was to test whether neural change predicted learning transfer and, if so, whether neural change offered predictive value over-and-above traditional performancebased assessments. We specifically sought to test, at the neural level, the MMT-based hypothesis that development of spatial resources-especially resources associated with spatial scanningpredicted improved verbal reasoning. We thus investigated whether neural changes on the spatial scanning task (EFT) predicted transfer to Reasoning. All models again controlled for PSAT, GPA, and Gender. Neural change on Reasoning was not considered in these analyses because the goal was to identify neural changes that could predict transfer to Reasoning. If the transfer task itself (Reasoning) is required in order to obtain a neural measure of transferable learning, then it is not clear that a meaningfully predictive inference can be made. Consistent with the MMT-based prediction, both of the spatial curriculum-related longitudinal changes in PPC activity identified for EFT ( $\triangle$ alPS_EFT and $\triangle \mathrm{IPL}$ EFT) were strongly associated with change in Reasoning performance ( $\Delta$ Reasoning; All $\beta>$.492, all $p<.001$; Tables S28-S29). That is, the more that spatial scanningrelated activity in alPS and IPL increased, the more students improved on verbal reasoning. Recall that, consistent with MMT, improved spatial scanning performance $(\Delta \mathrm{EFT})$ mediated the transfer association of the Geospatial curriculum to improved reasoning. We thus sought to mirror this analysis at the neural level, testing $\triangle$ alPS_EFT and $\triangle I P L \_E F T$ as neural mediators of transfer. Consistent with the behavioral findings, $\triangle$ alPS_EFT and $\triangle I P L$ EFT each mediated the transfer association of the Geospatial curriculum to $\Delta$ Reasoning (All Indirect Effects: $p<.001$; Fig. 3). The changes in EFT activity in prefrontal and premotor regions that were not associated with $\Delta \mathrm{EFT}$ (above) were also not associated with $\Delta$ Reasoning (All $\beta<.124$, all $p>.312$ ).

Having identified two curriculum-related changes in neural activity during spatial scanning (EFT) that predicted transfer to verbal reasoning, we next sought to compare these neural changes ( $\triangle$ alPS_EFT and $\triangle I P L \_E F T$ ) to performance-based assessments. To enable a direct comparison of the predictive value derived from neural change vs. performance-based learning assessment, 


\section{PREPRINT OF UNPUBLISHED MANUSCRIPT}

we compared EFT neural change vs. performance-based learning on the same EFT task ( $\triangle E F T)$. $\Delta$ EFT represented curriculum learning (specifically, the curriculum-related improvement in spatial scanning). As described above, $\Delta \mathrm{EFT}$ was also highly predictive of improvement in the Reasoning transfer task ( $\Delta$ Reasoning), which was the outcome variable we sought to predict in the current analysis. Thus, $\triangle E F T$ represented a strong performance-based predictor against which to compare neural change variables. Performance change on another curriculum-related learning outcome (Spatial Habits-Of-Mind;24, 30) was also included as a predictor of transfer, as were change in MRT performance ( $\triangle \mathrm{MRT}$ ), traditional academic assessments (PSAT and GPA), and Gender. Additionally, Geospatial course grades were included for models considering Geospatial students only. Including these multiple performance-based measures, in addition to $\Delta \mathrm{EFT}$, was intended to further increase the rigor of the comparison to neural change, and to reflect that the standard for demonstrating the value of neuroimaging predictors should be comparison to multiple performancebased tasks (not just one task) because of the greater pragmatic burden required for obtaining neuroimaging measures.

Multiple regression models ( $S I$ Materials and Methods) found that: 1) the two neural change predictor variables ( $\triangle$ alPS_EFT and $\triangle \mathrm{IPL} E E F T$ ) each individually yielded significantly better model fit for predicting $\triangle$ Reasoning than any of the performance-based assessments $(\triangle E F T, \triangle M R T$, change in Spatial Habits-Of-Mind, PSAT, GPA, Geospatial course grade [Geospatial students only], Gender; Likelihood Ratio Tests: all $p<.001)$; 2) the combination of $\Delta$ alPS_EFT and $\triangle \mathrm{IPL}$ EFT yielded significantly better fit than the best-fitting combinations of performance-based assessments (in the full neuroimaging sample: $\triangle E F T, \triangle M R T$, and PSAT; in Geospatial students only: $\Delta \mathrm{EFT}, \Delta \mathrm{MRT}$, and Geospatial course grades; all $p<.007$; Tables S45-S46); and 3) adding $\triangle$ alPS_EFT and $\triangle \mathrm{IPL} E F F T$ to the best-fitting combination of performance-based assessments in the full and Geospatial-only neuroimaging samples significantly improved fit (All $p<.043$; Table S49 and S52), and adding either $\triangle$ alPS_EFT or $\triangle I$ IPL_EFT individually improved fit for the full sample (All $p<.002$; Tables S47-48) and for the Geospatial-only sample in the case of $\triangle$ alPS_EFT $(p=.021$; Table S50) but not $\Delta \mathrm{IPL}$ EFT ( $p=.066$; Table S50). Notably, although the neural change variables were the strongest individual predictors, the strongest fit among all models was achieved by a model combining the two neural change variables ( $\triangle$ alPS_EFT and $\triangle \mathrm{IPL} E F T$ ) with three performance-based assessments ( $\triangle \mathrm{EFT}, \Delta \mathrm{MRT}$, PSAT; Adjusted $R^{2}=.39$; Table S53).

We further employed data-driven ensemble modeling prediction analysis(45; SI Materials and Methods), to identify the predictor variables that are most likely, in general, to be predictive of the outcome variable (in this case $\Delta$ Reasoning); that is, the variables that contribute the most to prediction across the possible models represented by the ensemble, rather than within an individual model. We first employed an ensemble model "boosting" analysis intended to optimize model accuracy (SI Materials and Methods;46), again including $\triangle$ alPS_EFT and $\Delta I P L \_E F T$ as the neural predictors, along with $\triangle E F T$ and the same set of additional performance-based predictors $(\triangle E F T$, $\triangle \mathrm{MRT}$, change in Spatial Habits-Of-Mind, PSAT, GPA, Geospatial course grade [Geospatial students only], Gender). The prediction analysis identified the two neural change predictor variables as the variables that contributed most to prediction of $\triangle$ Reasoning (Table S33): $\triangle \mathrm{IPL} E F T$ (accounting for $38 \%$ of predictor "importance") and $\triangle$ alPS_EFT (accounting for $28 \%$ ). Of the performance-based assessment variables, $\Delta$ EFT was assigned the highest predictor importance (16\%) across the ensemble model. When Geospatial course grade was included as a predictor (Geospatial students only) the model again indicated $\triangle$ alPS_EFT (26\%) and $\triangle \mathrm{IPL} E$ EFT (24\%) as the most important predictors, with $\triangle \mathrm{EFT}$ next at $18 \%$ (Table S35). Additional ensemble modeling analyses to build "standard" prediction models (Tables S33-44) largely converged with the boosting analyses. That is, data-driven variable selection resulted in the inclusion of neural change predictors in the most informative model (Akaike Information Criterion). Specifically, three variables were selected for inclusion in the standard prediction model: $\triangle \mathrm{IPL}$ EFT, PSAT, and $\triangle$ aIPS_EFT 


\section{PREPRINT OF UNPUBLISHED MANUSCRIPT}

(SI Materials and Methods). Iterating this analysis with Geospatial course grades included (Geospatial students only) resulted in the selection of two variables: $\triangle$ alPS_EFT and PSAT.

Lastly, post-hoc exploratory analyses investigated whether the longitudinal neural changes that were associated with improved reasoning performance were also associated with each other. As described above, the change in SpatialMap-to-DLPFC connectivity was the only longitudinal neural change during the Reasoning task that was significantly associated with improved reasoning performance. As also described above, changes in two PPC regions ( $\triangle$ alPS_EFT and $\triangle \mathrm{IPL} E \mathrm{EFT}$ ) predicted improved reasoning performance. We thus sought to test whether $\triangle$ alPS_EFT and $\triangle \mathrm{IPL} E F T$ were related to change in SpatialMap-to-DLPFC connectivity during Reasoning. The $\triangle$ alPS_EFT and $\triangle \mathrm{IPL} E F F T$ clusters spatially overlapped (in IPS and IPL) with areas of increased SpatialMap-to-DLPFC connectivity during Reasoning, and both $\triangle$ alPS_EFT $(\beta=.414, p<.001$; Table S55) and $\triangle \mathrm{IPL}$ EFT ( $\beta=.262, p=.038$; Table S56) predicted the increase in SpatialMap-toDLPFC connectivity. This post-hoc finding provides an additional indication that spatial education may influence IPS and IPL function in ways that support both spatial scanning and verbal reasoning (putatively because the same spatial attentional resources that support spatial scanning may also contribute to verbal reasoning, including via communication with DLPFC). Relatedly, and also posthoc, we tested whether the longitudinal changes in alPS activity that we observed separately in EFT and Reasoning were associated with each other, and found that they were $(\beta=.340, p=.006$; Table S57).

\section{Discussion}

Spatially-based accounts of human cognition (e.g., mental model theory;11, 47) lead to the educational hypothesis that fostering improvement in spatial cognitive processes might improve abilities beyond the spatial domain, including verbal reasoning. Consistent with this prediction, spatial education in the present study transferred to an untrained verbal reasoning task that is strongly linked to mental modeling. Further, the more students improved on spatial scanning and mental rotation-abilities specifically theorized to support mental modeling(12)-the more they improved on verbal reasoning, and improvement on spatial scanning mediated the association of the spatial curriculum to improved verbal reasoning. Likewise, at the neural level, longitudinal fMRI detected spatial curriculum-related increases in the activity of spatial cognition-implicated brain regions (aIPS and IPL) during spatial scanning (EFT). These neural changes were associated with improvement in spatial scanning performance, and mediated the association of the spatial curriculum to verbal reasoning. These findings experimentally extend evidence for mental modeling as a basis of human reasoning (10)-to our knowledge, no prior work has shown that fostering gains in spatial ability is associated with gains in verbal reasoning-and indicate that MMT can be translated to real-world STEM classrooms via spatial education. The present evidence suggests further exploration of spatial scanning as a trainable ability to support reasoning in educational and other contexts.

Addressing research priorities noted in the NRC, OECD, and AEI reports (19, 20, 23), the present work shows evidence for the real-world efficacy of an in-school implementation of spatial education, and empirically indicates plausible cognitive and neural mechanisms-of-change. With respect to efficacy, improved spatial scanning, spatial habits-of-mind (another outcome strongly linked to STEM achievement;30), and verbal reasoning represent spatial curriculum-driven gains both within and beyond the spatial domain. This evidence was strengthened by the use of propensity scoring methods to reduce selection bias. Regarding mechanisms-of-change, the above-noted findings implicate curriculum-related cognitive and neural changes in spatial scanning as plausible mechanisms. Additional evidence indicated that SpatialMap connectivity to a reasoning-associated DLPFC region increased longitudinally in Geospatial students relative to Controls. The amount of increase in this connectivity predicted the amount of improvement in reasoning. These findings point to increased SpatialMap-to-DLPFC communication as a neural change that may enable better 


\section{PREPRINT OF UNPUBLISHED MANUSCRIPT}

reasoning, putatively because this communication facilitates greater contribution of spatial resources to reasoning (e.g., spatial attentional resources linked to IPL and IPS). Female students showed greater curriculum-driven increases in connectivity than males, suggesting that this connectivity may be especially important for the effects of spatial education in girls. This is noteworthy with respect to previous evidence indicating the potential of spatial education to support female students' participation and achievement in STEM(48), particularly given the relationship of both spatial abilities and reasoning to STEM achievement(21). Additionally, evidence that the spatial curriculum was associated with changes in activity and representational similarity in IPS during reasoning-even though these changes were not associated with task performance-further suggests that spatial education influences the function of IPS, which is associated with diverse spatial cognition-related abilities $(35,38-40)$. In the context of growing evidence and enthusiasm for the importance of spatial cognition among education researchers $(19,20,47)$, which has not yet permeated education policy and practice $(19,20,23)$, the classroom-based evidence obtained in the present study provides new empirical support for the adoption of spatial education.

The present research shows proof-of-principle for using in-school neural change to predict learning transfer more accurately than traditional performance-based assessments. We predicted improvement on the Reasoning transfer task from neural change on a separate task (EFT; reflecting the MMT-based hypothesis that spatial scanning supports mental model reasoning;12). Direct model comparisons, and ensemble modeling prediction analysis, showed that EFT neural changes in IPL and IPS were stronger predictors of transfer than performance-based academic and cognitive assessments (even when comparing neural vs. performance change on the same EFT task). Notably, however, the best prediction of learning transfer was achieved by combining neural changes with traditional assessments. These findings indicate that neural change has the capacity to improve assessment of the transferability of curricula, and suggest that the most effective approaches will likely employ measures of neural change to bolster (but not replace) traditional assessments.

Assessing the transferability of curricula used for classroom teaching can be accomplished on relatively small scales-including the small-scale development of the Geospatial curriculum by researchers and high school educators in Virginia(24). Nonetheless, the curricula that emerge from such assessment efforts can have broad impacts(5-7). Leveraging neural changes that predict transferability to enable neurally-informed curriculum assessment thus has long-term promise as a means by which neuroscience may inform education by informing curriculum design (e.g., by identifying existing curricula that are likely to achieve transfer, identifying changes to curricula that can improve transfer, and informing development of novel curricula), while avoiding the practical and ethical limitations of large-scale neuroimaging (by assessing curricula rather than individual students). At a more basic implementation level, fMRI detection of curriculum-specific, in-school longitudinal changes in neural activity, connectivity, and representational similarity in the present study supports the efficacy of longitudinal fMRI to study learning of specific curricula in real-world classroom environments.

While neural change-based prediction of learning transfer suggests future educational applications, determining how broad or specific such applications might be will require investigating the breadth of transferability that neural changes-especially in spatial cognition-related regions-can predict. Whereas this proof-of-principle study focused on spatial scanning in EFT (near transfer), and more distant transfer to verbal Reasoning, it seems unlikely that the curriculum-related neural changes observed here would uniquely influence these two tasks. Testing wider arrays of transfer tasks to determine the extent to which neural changes reflect development of underlying abilities (i.e., abilities that are not task-specific) can determine the more global value of neuroimaging-based transfer prediction. Neural changes in spatial cognition-implicated brain regions are plausible candidates to predict broad transferability. Transfer from spatial training to math has been recently observed(49), and spatial resources-including spatial attentional resources associated with IPL 


\section{PREPRINT OF UNPUBLISHED MANUSCRIPT}

and IPS-appear to support diverse forms of cognition (e.g., planning, creativity, numeracy, causal understanding, linguistic representation), many of which are linked to mental modeling $(10,44,50)$.

\section{Materials and Methods \\ The Geospatial Course}

The Geospatial Course(24) was created in 2005 as a partnership between public high schools in Virginia and the Integrated Science and Technology Department at James Madison University (JMU). Students take the course for either 45 minutes daily or 90 minutes every other day, and receive college credit from JMU. JMU faculty mentor the high school teachers, providing technical support, teaching and observing classes, and assisting in mentoring students on their course projects. The Geospatial Course curriculum is designed to enhance spatial thinking skills, including spatial scanning, and foster increased use of spatial thinking strategies through the use of geospatial technology such as Geographic Information Systems (e.g., ArcGIS; www.arcgis.com). More information about the Geospatial Course is provided at https://www.isat.jmu.edu/geospatialsemester/.

\section{Behavioral Tasks}

\section{Reasoning}

On each Reasoning trial(33), participants indicated by keypress ("Yes"/"No") whether a conclusion sentence followed logically from two preceding premise sentences. Of 60 total trials, 40 were reasoning problems, 20 involving spatial relations (e.g., "above"/"below") and 20 involving nonspatial relations (e.g., "better"/"worse"). The remaining 20 trials were matching problems, which required participants to determine whether the conclusion exactly matched either of the two premises. Following Ruff et al. (33), these matching items served as a control condition for neuroimaging analyses, and were not included in behavioral performance analysis. For each trial type, half were True and half were False. Once the conclusion sentence appeared, participants had up to 8 seconds to respond (SI Materials and Methods; Fig. S3 displays complete timing). Behavioral performance scores for the Reasoning task (and all behavioral tasks) were computed as the number of correct responses per second (rate correct score;51; SI Materials and Methods). Table S8 displays descriptive statistics for Reasoning. Results of Group(Geospatial, Control)-byTime $(\mathrm{T} 1, \mathrm{~T} 2)$ ANOVA are shown in Table S3.

\section{Embedded Figures Task (EFT)}

On each EFT trial (35; Fig. S2), participants were given up to 10 seconds to respond by keypress ("Yes"/"No") to indicate whether a simple figure could be found within a more complex figure. There were 30 total trials (20 True, 10 False). Task trials were compared to Baseline Fixation in the fMRI analyses, as in previous implementations(52). Descriptive statistics for EFT are shown in Table S7. Results of Group(Geospatial, Control)-by-Time(T1, T2) ANOVA are shown in Table S2.

\section{Mental Rotation Task (MRT)}

On each MRT trial(28, 36; Fig. S4) participants were given up to 7 seconds to indicate by keypress ("Yes"/"No") whether two images depicted rotations of the same 3-dimensional object. Of 84 total trials, each of three different angles of rotation (50,100, 150 degrees) was used 24 times. Following Voyer et al.(53), we used a 2:1 ratio of True (same object) to False (different objects) trials across all trial types. Consistent with prior work(43), fMRI analysis contrasted True trials vs. control trials (0 degrees of rotation). Descriptive statistics for MRT are shown in Table S9. Results of Group(Geospatial, Control)-by-Time(T1, T2) ANOVA are shown in Table S5. 


\title{
PREPRINT OF UNPUBLISHED MANUSCRIPT
}

\author{
Spatial Habits-of-Mind Inventory (SHOMI)
}

The SHOMI(30) is a 28-items self-report survey assessing the extent to which individuals engage in essential dimensions of spatial thinking strategy use, including pattern recognition, spatial description, visualization, and spatial concept use. Participants rate (1-5) how well statements about spatial thinking strategy use apply to them, and a total sum score is calculated (highest possible score $=140$ ). While other behavioral measures were administered at T1 and T2, SHOMI was administered at pre-test and T2. Descriptive statistics for SHOMI are shown in Table S10. Results of Group(Geospatial, Control)-by-Time(T1, T2) ANOVA are shown in Table S6.

\section{Statistical Analysis}

All statistical analyses were computed in R-studio(54) and SPSS Version 27(46). Ensemble modeling prediction analyses were computed via Automatic Linear Modeling in SPSS 27(45). See SI Materials and Methods for more detailed descriptions of analyses.

\section{fMRI Data Acquisition and Analysis}

Imaging acquisition was performed on a Siemens 3T TIM Trio MRI scanner. All task fMRI data were acquired from T2*-weighted echoplanar imaging sequences $(373.0 \mathrm{~mm}$ transversal slices; $64 \times 64$ matrix; repetition time $=2000 \mathrm{~ms}$; echo time $=30 \mathrm{~ms}$; field of view $=192 \mathrm{~mm} ; 3.0 \times 3.0 \times 3.0 \mathrm{~mm}$ voxels; flip angle $=90^{\circ}$ ). To account for magnet stabilization, the first 2 volumes were excluded from analysis. High-resolution T1-weighted anatomical images (176 $1.00 \mathrm{~mm}$ slices; 256x256 matrix; repetition time $=1900 \mathrm{~ms}$; echo time $=2.52 \mathrm{~ms}$; field of view $=250 \mathrm{~mm} ; 1.0 \times 1.0 \times 1.0 \mathrm{~mm}$; flip angle $=90^{\circ}$ )

fMRI data preprocessing was carried out using FEAT (fMRI Expert Analysis Tool) Version 5.98, part of FSL (FMRIB's Software Library); preprocessing steps included spatial smoothing, motion correction, intensity normalization, temporal filtering, straight line fitting(55)

Whole-brain activation analysis for each task was conducted using standard GLM-based subjectlevel and group-level analyses in FSL (including Group-by-Time ANOVA; SI Materials and Methods). Region of Interest (ROI) analysis were conducted using FSL's featquery tool (http://www.fmrib.ox.ac.uk/fsl/feat5/featquery.html) to extract mean activation levels in clusters of interest.

Functional connectivity analysis employed a standard PPI procedure(56). An a priori DLPFC seed region was constructed as a $10 \mathrm{~mm}$ sphere around a meta-analytically identified peak voxel associated with syllogistic reasoning(11).

Representational similarity analysis used the fslstats tool to extract mean activation levels from each voxel in the alPS cluster during three conditions: spatial reasoning, nonspatial reasoning, and matching (control). Each condition was considered as a contrast to baseline fixation (e.g., spatial reasoning $>$ fixation) to reduce the influence of elements of no interest. Pearson's partial correlations were then computed between voxel activity during spatial reasoning and nonspatial reasoning, controlling for the matching condition. Because $r$ values are non-normally distributed, $r$ values were next transformed using Fisher's z-transformation. All relevant statistics were then computed using these $z$ values as inputs for each participant at T1 and T2.

\section{Acknowledgments}

We thank the teachers and school administrators at each of the participating high schools for their partnership and insights, and the students and their families for their patience and commitment. 


\section{PREPRINT OF UNPUBLISHED MANUSCRIPT}

We also thank Sangeet Khemlani for comments on a previous version of the manuscript, and Anna Johnson, Vivian Wong, and Jaeil Ahn for methodological consultation.

\section{Funding}

This work was supported by grants from the National Science Foundation to A.E.G., R.A.K, D.H.U., and D.J.M.K (DRL-1420481, EHR-1661065), and A.E.G. (EHR-1920682). R.A.C.'s research is supported by a National Science Foundation Graduate Research Fellowship.

Data and materials availability

All data are fully available on the Open Science Framework (https://osf.io/kfjir4/). 


\section{PREPRINT OF UNPUBLISHED MANUSCRIPT}

\section{References}

1. J. D. E. Gabrieli, The promise of educational neuroscience: Comment on Bowers (2016). Psychol. Rev. 123, 613-619 (2016).

2. D. T. Willingham, J. W. Lloyd, How Educational Theories Can Use Neuroscientific Data. Mind, Brain, Educ. 1, 140-149 (2007).

3. S. Dehaene, How we learn: Why brains learn better than any machine...for now (Penguin, 2021).

4. S. M. Barnett, S. J. Ceci, When and where do we apply what we learn?: A taxonomy for far transfer. Psychol. Bull. 128 (2002), pp. 612-637.

5. D. L. Cusumano, Is it working?: An overview of curriculum based measurement and its uses for assessing instructional, intervention, or program effectiveness. Behav. Anal. Today. 8, 24-34 (2007).

6. J. M. Voogt, J. M. Pieters, A. Handelzalts, Teacher collaboration in curriculum design teams: effects, mechanisms, and conditions. Educ. Res. Eval. 22, 121-140 (2016).

7. B. Garelick, Miracle Math: A Successful Program from Singapore Tests the Limits of School Reform in the Suburbs. Educ. Next. 6, 38-45 (2006).

8. S. A. Bunge, E. R. Leib, How Does Education Hone Reasoning Ability? Curr. Dir. Psychol. Sci. 29, 167-173 (2020).

9. M. Meshulam, L. Hasenfratz, H. Hillman, Y.-F. Liu, M. Nguyen, K. A. Norman, U. Hasson, Neural alignment predicts learning outcomes in students taking an introduction to computer science course. Nat. Commun. 12, 1922 (2021).

10. P. N. Johnson-Laird, Mental models and human reasoning. Proc. Natl. Acad. Sci. U. S. A. 107, 18243-18250 (2010).

11. I. Kant, Critique of Pure Reason (1781).

12. M. Knauff, in Spatial Cognition \& Computation (ed. 9, 2009), pp. 109-137.

13. P. N. Johnson-laird, P. N. Johnson-laird, Mental models, deductive reasoning and the brain (1995) (available at http://citeseerx.ist.psu.edu/viewdoc/summary?doi=10.1.1.174.4386).

14. J. Prado, A. Chadha, J. R. Booth, The Brain Network for Deductive Reasoning: A Quantitative Meta-analysis of 28 Neuroimaging Studies. J. Cogn. Neurosci. 23, 34833497 (2011).

15. C. Wendelken, E. Ferrer, K. J. Whitaker, S. A. Bunge, Fronto-Parietal Network Reconfiguration Supports the Development of Reasoning Ability. Cereb. Cortex. 26, 2178-2190 (2016).

16. A. P. Mackey, A. T. Miller Singley, S. A. Bunge, Intensive Reasoning Training Alters Patterns of Brain Connectivity at Rest. J. Neurosci. 33, 4796-4803 (2013).

17. R. E. Jung, R. J. Haier, The Parieto-Frontal Integration Theory (P-FIT) of intelligence: Converging neuroimaging evidence. Behav. Brain Sci. 30, 135-154 (2007). 
PREPRINT OF UNPUBLISHED MANUSCRIPT

18. V. Goel, R. J. Dolan, Functional neuroanatomy of three-term relational reasoning. Neuropsychologia. 39, 901-909 (2001).

19. National Research Council, Learning to think spatially. (National Academies Press, 2006).

20. N. S. Newcombe, "Harnessing spatial thinking to support stem learning" (Organisation for Economic Cooperation and Development, 2017).

21. J. Wai, D. Lubinski, C. P. Benbow, Spatial ability for STEM domains: Aligning over 50 years of cumulative psychological knowledge solidifies its importance. J. Educ. Psychol. 101, 817-835 (2009).

22. D. H. Uttal, D. I. Miller, N. S. Newcombe, Exploring and Enhancing Spatial Thinking: Links to Achievement in Science, Technology, Engineering, and Mathematics? Curr. Dir. Psychol. Sci. 22, 367-373 (2013).

23. J. Wai, D. H. Uttal, Why spatial reasoning matters for education policy. Am. Enterp. Inst. (2018).

24. B. Kolvoord, K. Keranen, S. Rittenhouse, The Geospatial Semester: Concurrent Enrollment in Geospatial Technologies. J. Geog. 118, 3-10 (2019).

25. S. Dehejia, R. H. \& Wahba, Propensity score-matching methods for nonexperimental causal studies. Rev. Econ. Stat. 84, 151-161 (2002).

26. S. O. Becker, A. Ichino, Estimation of Average Treatment Effects Based on Propensity Scores. Stata J. Promot. Commun. Stat. Stata. 2, 358-377 (2002).

27. H. A. Witkin, Individual differences in ease of perception of embedded figures. J. Pers. 19, $1-15$ (1950).

28. R. Shepard, J. Metzler, Mental rotation of three-dimensional objects. Science. 171, 701-3 (1971).

29. H. R. Liesefeld, M. Janczyk, Combining speed and accuracy to control for speed-accuracy trade-offs(?). Behav. Res. Methods. 51, 40-60 (2019).

30. M. Kim, R. Bednarz, Effects of a GIS Course on Self-Assessment of Spatial Habits of Mind (SHOM). J. Geog. 112, 165-177 (2013).

31. M. Caliendo, S. Kopeinig, Some Practical Guidance For the Implementation of Propensity Score Matching. J. Econ. Surv. 22, 31-72 (2008).

32. D. Voyer, S. Voyer, M. P. Bryden, Magnitude of sex differences in spatial abilities: A metaanalysis and consideration of critical variables. Psychol. Bull. 117, 250-270 (1995).

33. C. C. Ruff, M. Knauff, T. Fangmeier, J. Spreer, Reasoning and working memory: common and distinct neuronal processes. Neuropsychologia. 41, 1241-1253 (2003).

34. P. N. Johnson-Laird, Mental models and deduction. Trends Cogn. Sci. 5, 434-442 (2001).

35. E. Walter, P. Dassonville, Activation in a Frontoparietal Cortical Network Underlies Individual Differences in the Performance of an Embedded Figures Task. PLoS One. 6, e20742 (2011).

36. M. Peters, C. Battista, Applications of mental rotation figures of the Shepard and Metzler 


\section{PREPRINT OF UNPUBLISHED MANUSCRIPT}

type and description of a mental rotation stimulus library. Brain Cogn. 66, 260-264 (2008).

37. T. Yarkoni, R. A. Poldrack, T. E. Nichols, D. C. Van Essen, T. D. Wager, Large-scale automated synthesis of human functional neuroimaging data. Nat. Methods. 8, 665-670 (2011).

38. C. R. Gillebert, D. Mantini, V. Thijs, S. Sunaert, P. Dupont, R. Vandenberghe, Lesion evidence for the critical role of the intraparietal sulcus in spatial attention. Brain. 134, 1694-1709 (2011).

39. K. L. Alfred, A. C. Connolly, J. S. Cetron, D. J. M. Kraemer, Mental models use common neural spatial structure for spatial and abstract content. Commun. Biol. 3, 1-11 (2020).

40. J. T. Coull, C. D. Frith, Differential Activation of Right Superior Parietal Cortex and Intraparietal Sulcus by Spatial and Nonspatial Attention. Neuroimage. 8, 176-187 (1998).

41. J. C. Lynch, V. B. Mountcastle, W. H. Talbot, T. C. Yin, Parietal lobe mechanisms for directed visual attention. J. Neurophysiol. 40, 362-389 (1977).

42. C. D. Chambers, J. M. Payne, M. G. Stokes, J. B. Mattingley, Fast and slow parietal pathways mediate spatial attention. Nat. Neurosci. 7, 217-218 (2004).

43. J. M. Zacks, Neuroimaging Studies of Mental Rotation: A Meta-analysis and Review. J. Cogn. Neurosci. 20, 1-19 (2008).

44. M. Piazza, V. Izard, P. Pinel, D. Le Bihan, S. Dehaene, Tuning Curves for Approximate Numerosity in the Human Intraparietal Sulcus. Neuron. 44, 547-555 (2004).

45. H. Yang, The case for being automatic: introducing the automatic linear modeling (LINEAR) procedure in SPSS statistics. Mult. Linear Regres. Viewpoints. 39, 27-37 (2013).

46. I. Corp, IBM SPSS Statistics for Windows, Version 27.0 (2020).

47. D. H. Uttal, N. G. Meadow, E. Tipton, L. L. Hand, A. R. Alden, C. Warren, N. S. Newcombe, The malleability of spatial skills: A meta-analysis of training studies. Psychol. Bull. 139, 352-402 (2013).

48. L. S. Liben, The STEM gender gap: The case for spatial interventions. Int. J. Gender, Sci. Technol. 7, 133-150 (2015).

49. N. Judd, T. Klingberg, Training spatial cognition enhances mathematical learning in a randomized study of 17,000 children. Nat. Hum. Behav. (2021), doi:10.1038/s41562-02101118-4.

50. D. Gentner \& A.L. Stevens, Mental models (Psychology Press, 2014).

51. A. Vandierendonck, A comparison of methods to combine speed and accuracy measures of performance: A rejoinder on the binning procedure. Behav. Res. Methods. 49, 653-673 (2017).

52. S. R. Damarla, T. A. Keller, R. K. Kana, V. L. Cherkassky, D. L. Williams, N. J. Minshew, M. A. Just, Cortical underconnectivity coupled with preserved visuospatial cognition in autism: Evidence from an fMRI study of an embedded figures task. Autism Res. 3, 273279 (2010). 


\section{PREPRINT OF UNPUBLISHED MANUSCRIPT}

53. D. Voyer, J. Hou, Type of items and the magnitude of gender differences on the Mental Rotations Test. Can. J. Exp. Psychol. Can. Psychol. expérimentale. 60, 91-100 (2006).

54. J. Verzani, Using R for Introductory Statistics (Chapman and Hall/CRC, 2018; https://www.taylorfrancis.com/books/9781315362670).

55. S. M. Smith, M. Jenkinson, M. W. Woolrich, C. F. Beckmann, T. E. J. Behrens, H. Johansen-Berg, P. R. Bannister, M. De Luca, I. Drobnjak, D. E. Flitney, R. K. Niazy, J. Saunders, J. Vickers, Y. Zhang, N. De Stefano, J. M. Brady, P. M. Matthews, in Neurolmage (Academic Press, 2004), vol. 23, pp. S208--S219.

56. J. X. O'Reilly, M. W. Woolrich, T. E. J. Behrens, S. M. Smith, H. Johansen-Berg, Tools of the trade: psychophysiological interactions and functional connectivity. Soc. Cogn. Affect. Neurosci. 7, 604-609 (2012). 


\section{Figures and Tables}

A

T1

Before school year Complete all behavioral measures (Reasoning, EFT and MRT during scan for fMRI group)

B
Full Academic Year

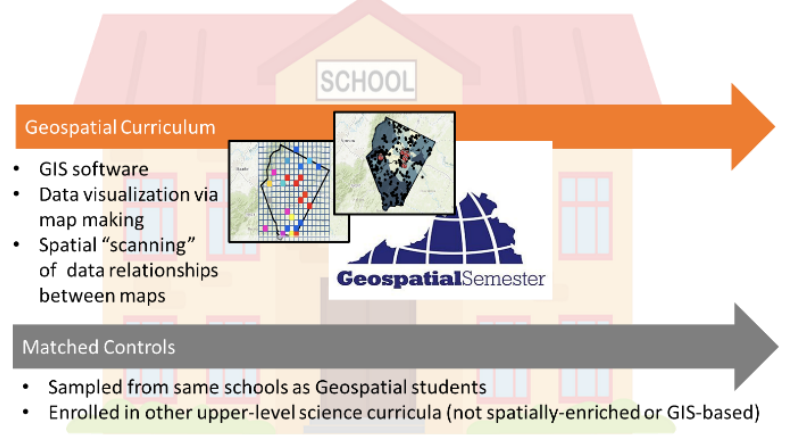

Embedded Figures (EFT) Mental Rotation (MRT)
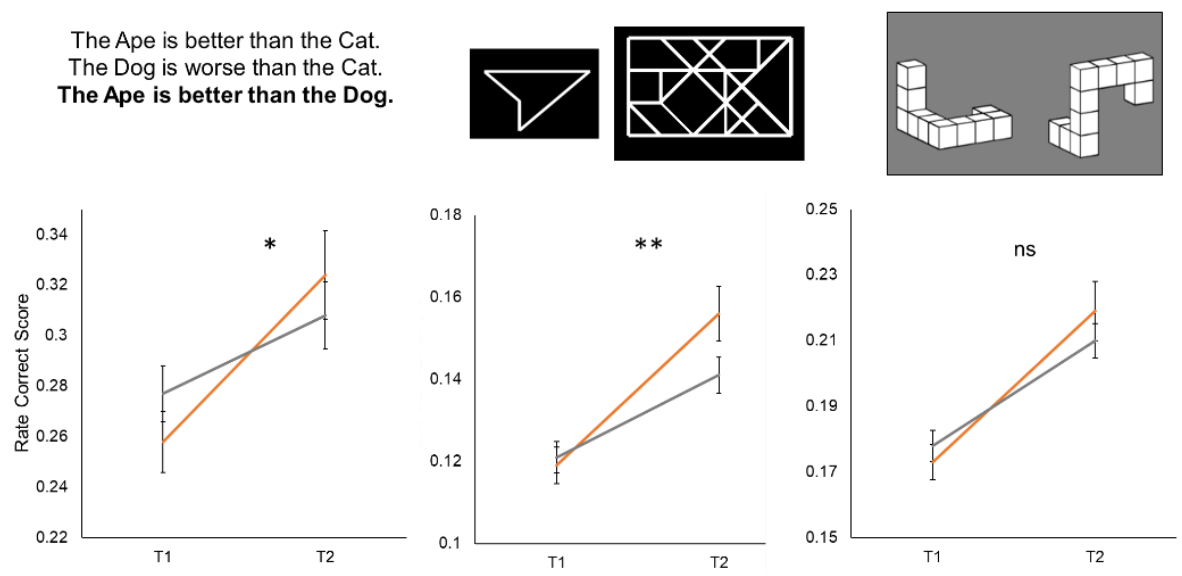
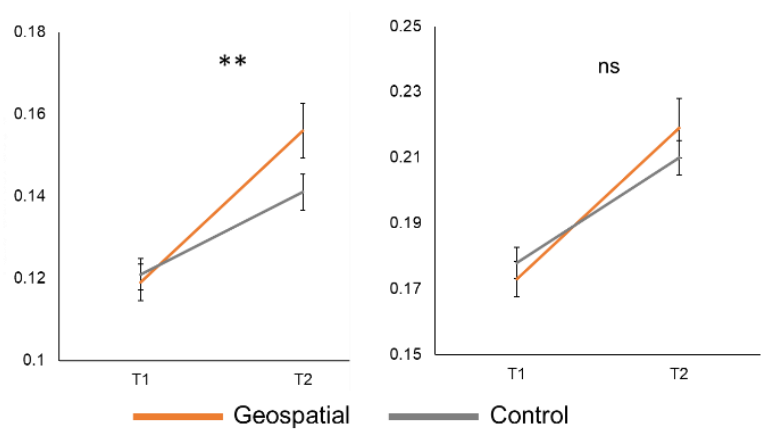

Control
The Ape is better than the Cat. The Dog is worse than the Cat. The Ape is better than the Dog.
T2

After school year Complete all behavioral measures (Reasoning, EFT and MRT during scan for fMRI group)
Spatial Habits of Mind

"I find that graphs, charts, or maps help me learn new concepts."

"Using spatial terms enables me to describe certain things more efficiently and effectively."

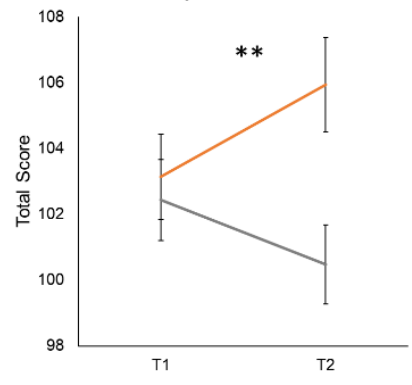

Figure 1. Study design and transfer results. Panel A depicts the longitudinal (pre-post) quasiexperimental in-school design comparing Geospatial students to matched Controls at the same high schools. The paired map images representing the Geospatial curriculum (called "Geospatial Semester") are an example GIS-based visualization of spatial data relationships, taken from a student project mapping the distribution of high-speed internet resources within a geographic region. Panel $B$ shows example stimuli for tasks administered before and after the school year, and longitudinal performance change for these tasks $\left({ }^{*} p<.05,{ }^{* *} p<.01\right)$. Alternate versions of Reasoning, EFT, and MRT, were counterbalanced across T1 and T2. The Spatial Habits-of-Mind inventory was administered at pre-test (prior to T1) and at T2. 
PREPRINT OF UNPUBLISHED MANUSCRIPT

Curriculum-related change in activity during Reasoning in alPS

A

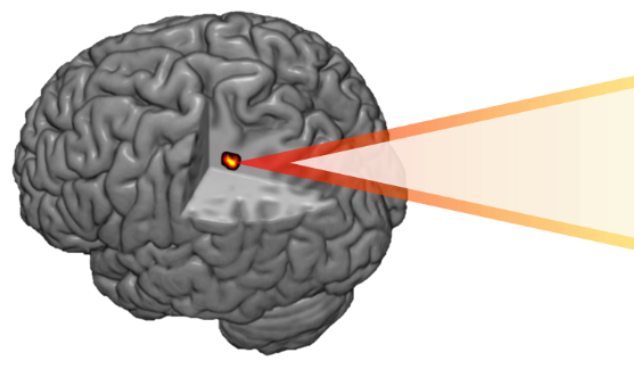

Curriculum-related change in alPS neural similarity for Reasoning (spatial vs. nonspatial relations)

B

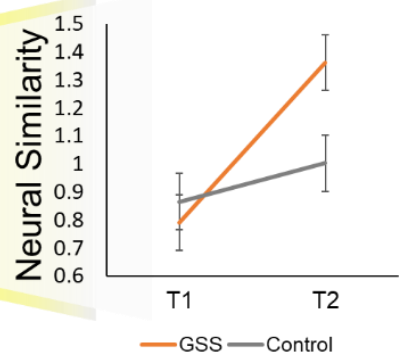

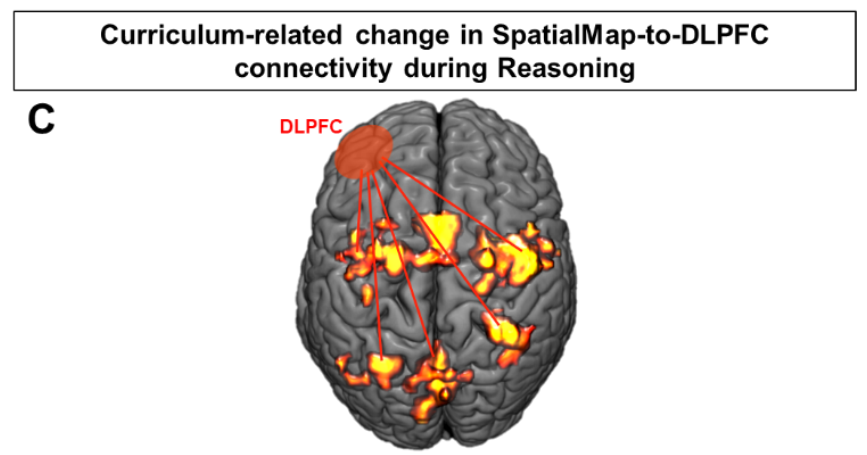

Figure 2. Longitudinal neural changes during Reasoning. Geospatial students showed greater longitudinal increase in alPS activity within Neurosynth-based SpatialMap (A), increased representational similarity between spatial and nonspatial reasoning relations in this alPS cluster (B), and increased connectivity of SpatialMap to DLPFC region meta-analytically implicated in syllogistic deductive verbal reasoning $(14 ; \mathrm{C})$. 
A

\section{EFT}

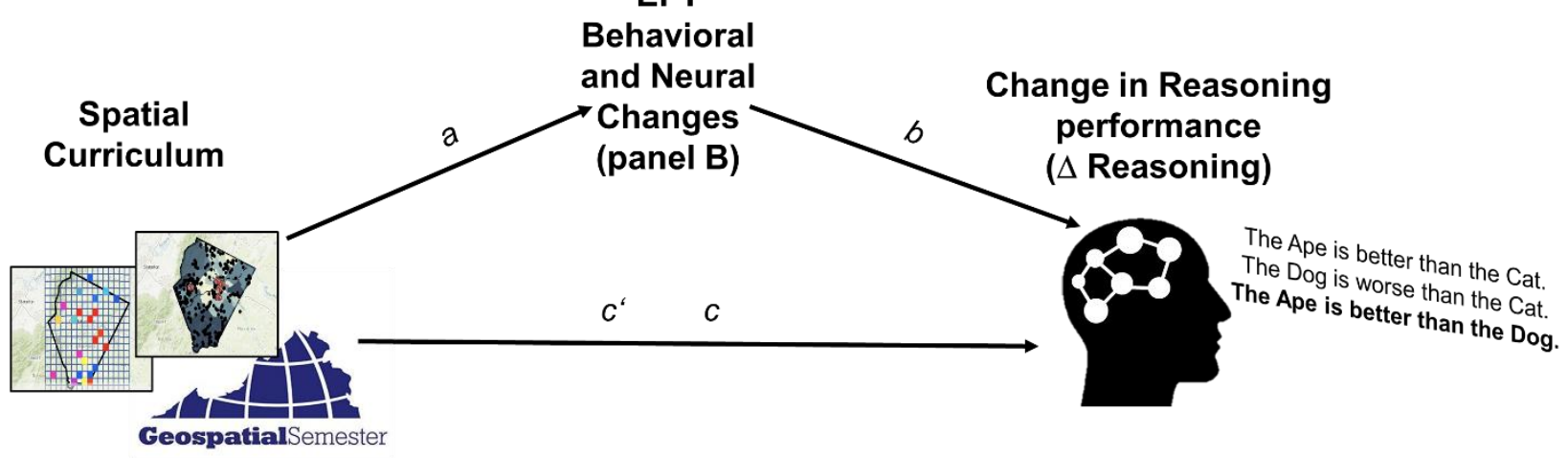

B

$\triangle$ EFT Behavioral

$\Delta$ Activity in alPS during EFT

$\triangle$ Activity in IPL

Performance

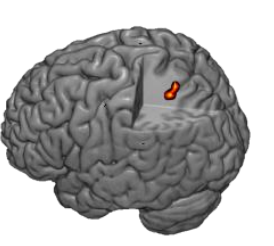
during EFT
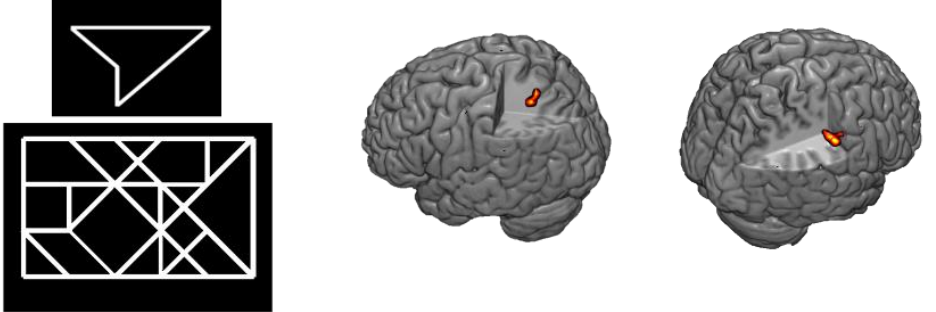

\begin{tabular}{|l|l|l|l|}
\hline $\mathrm{a}$ & $.177^{*}$ & $.377^{*}$ & $.358^{*}$ \\
\hline $\mathrm{b}$ & $.288^{* * *}$ & $.492^{* * *}$ & $.581^{* * *}$ \\
\hline $\mathrm{c}^{\prime}(\mathrm{c})$ & $.135\left(.187^{*}\right)$ & $.221\left(.376^{* *}\right)$ & $.192\left(.376^{* *}\right)$ \\
\hline Indirect Effect & $\mathrm{P}=.028$ & $\mathrm{P}<.001$ & $\mathrm{P}<.001$ \\
\hline
\end{tabular}

Figure 3. Mediation of transfer by changes in spatial scanning. Panel A depicts mediation of transfer from the spatial curriculum to improved reasoning by the performance changes and neural changes on the spatial scanning task (EFT) that are shown in Panel B. The brain images in panel $B$ display clusters in alPS and IPL where Geospatial students showed greater longitudinal increases in activation than Controls during EFT. The table in Panel B displays the path coefficients and indirect effects for models with each of the three EFT change variables as mediators. 